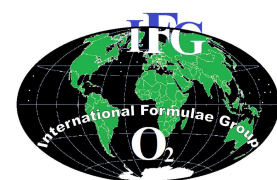

\title{
Etude ethnobotanique des légumes feuilles thérapeutiques utilisés dans le traitement des diarrhées au sud-Bénin (Afrique de l'Ouest)
}

\author{
A. J. AGBANKPÉ ${ }^{1,3}$, T. V. DOUGNON ${ }^{1,2 *}$, H. S. BANKOLE ${ }^{1,3}$, B. YÈHOUÉNOU ${ }^{3}$, \\ H. YEDOMONHAN ${ }^{4}$, M. LEGONOU ${ }^{3}$ et T. J. DOUGNON ${ }^{1}$
}

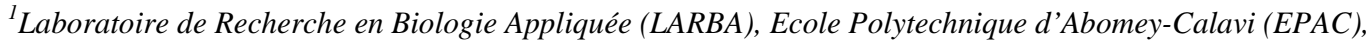 Université d'Abomey-Calavi (UAC), 01 BP 2009 Cotonou, Bénin.
${ }^{2}$ Laboratoire d'Hygiène-Assainissement, d'Ecotoxicologie et d'Environnement Santé (HECOTES), ex- Laboratoire de Toxicologie et de Santé Environnementale, Centre Interfacultaire de Formation et de Recherche en Environnement pour le Développement Durable (CIFRED), 01 BP 1463 Cotonou, Bénin. ${ }^{3}$ Laboratoire de Microbiologie Alimentaire, Ministère de la Santé, 01 BP 418 Cotonou, Bénin.
${ }^{4}$ Laboratoire de Botanique et d'Ecologie Végétale, FAST/ UAC, 01 BP 4521 Cotonou, Bénin.
Auteur correspondant; E-mail : victorien88@ hotmail.com ; Tel : 00 (229) 97736446.

\section{RESUME}

Les légumes-feuilles constituent d'excellents apports pour la diversification de l'alimentation humaine. La présente étude a permis d'identifier les légumes-feuilles à propriétés anti-diarrhéiques consommés à Cotonou et Abomey-Calavi (Sud-Bénin). A l'aide d'enquêtes basées sur la méthode d'Interview Semi-Structurée, il a été recensé vingt-sept (27) espèces de légumes feuilles regroupées en vingt-trois genres (23) et appartenant à dix-sept (17) familles botaniques. Les familles les plus représentées, en termes d'espèce, sont les Amaranthaceae et les Lamiaceae. Les espèces les plus consommées pour le traitement des infections diarrhéiques sont : Ocimum gratissimum L. (29,05\%), Vernonia amygdalina Delile. (16,80\%), Crateva adansonii DC. ssp adansonii $(13,49 \%)$ et Sesamum radiatum L. (11,41\%). Ces légumes-feuilles sont préparés à l'état frais ou séchés (poudres) puis consommés comme légume d'accompagnement (sauce) ou utilisés comme tisane. Ces résultats constituent la base d'études ultérieures visant à évaluer expérimentalement les potentialités antibactériennes et anti-diarrhéiques de ces légumes-feuilles. Cela permettra de mettre à la disposition des populations, des Médicaments Traditionnels Améliorés.

(C) 2014 International Formulae Group. All rights reserved.

Mots clés : Ethnobotanique, diarrhées, légumes feuilles, médecine traditionnelle.

\section{INTRODUCTION}

Dans les pays en développement, les infections entériques et les toxi-infections alimentaires constituent une préoccupation importante de santé publique à cause de leur fréquence et de leur gravité. En effet, elles sont à l'origine de plus de 17 millions de décès par an de par le monde dont plus de la

(C) 2014 International Formulae Group. All rights reserved. DOI : http://dx.doi.org/10.4314/ijbcs.v8i4.36 
moitié provient du continent africain (OMS, 2011). Ce problème important de santé publique touche tous les groupes d'âge et particulièrement les couches sensibles telles que les nourrissons, les enfants et les personnes âgées. Ces infections ont comme principal symptôme, des diarrhées qui peuvent se révéler très graves (Gagnon, 2007). Les maladies diarrhéiques représentent $20 \%$ de toutes les maladies dans les pays en voie de développement. Elles sont la deuxième cause de décès chez les enfants de moins de 5 ans. Elles représentent un véritable problème de santé publique dans le monde entier où elles sont responsables de 4 à 6 millions de décès par an (Bryce et al., 2005 ; Liu et al., 2012).

$\mathrm{Au}$ Bénin, $80 \%$ de la population utilisent encore la médecine traditionnelle pour se soigner, à l'instar de l'ensemble des pays en développement, (WHO, 2009). Parmi les ressources végétales utilisées, les légumes constituent des apports excellents d'enrichissement et de diversification de l'alimentation de l'homme. Il s'agit de véritables sources primaires de nutriments minéraux, de vitamines, de fibres et autres composés qui interviennent dans la santé humaine (Sinha, 2011 ; Dossou et al., 2012). Il est donc primordial de rechercher le potentiel médicinal de certains légumesfeuilles thérapeutiques consommés au Bénin. Ceci pour, en plus de la sécurité alimentaire, mettre à la disposition de la population des Médicaments Traditionnels Améliorés efficaces, peu onéreux et accessibles à tous.

C'est dans ce cadre que s'inscrit le présent travail dont l'objectif global est d'inventorier les légumes feuilles thérapeutiques consommés au sud-Bénin pour le traitement des diarrhées. De façon spécifique, il s'agit de :

- apprécier les caractéristiques socioculturelles des consommateurs et utilisateur de légumes feuilles au sud-Bénin ;

- recenser les principaux légumes feuilles thérapeutiques consommés au sud-Bénin pour le traitement des infections diarrhéiques y compris leurs données ethnobotaniques.

\section{MATERIEL ET METHODES Milieu d'étude}

Les enquêtes ethnobotaniques ont été menées dans les deux communes les plus peuplées de la République du Bénin : Cotonou et Abomey-Calavi. La zone d'étude est située entre $6^{\circ} 20^{\prime}$ et $6^{\circ} 43^{\prime}$ de latitude Nord et $2^{\circ} 12^{\prime}$ et $2^{\circ} 30^{\prime}$ de longitude Est (Figure 1). Elle couvre une superficie de 61.439 hectares pour une population de 1.334.839 habitants (INSAE, 2013). Le climat est de type subéquatorial avec l'alternance de deux saisons pluvieuses et deux saisons sèches. La pluviosité moyenne est de $1200 \mathrm{~mm}$ par an, avec une température moyenne annuelle variant de $26{ }^{\circ} \mathrm{C}$ à $28{ }^{\circ} \mathrm{C}$ et une humidité relative supérieure à $60 \%$ (Akoègninou, 2004).

\section{Méthodes}

\section{Collecte des données}

Des enquêtes basées sur la méthode d'Interview Semi-Structurée (Dibong et al., 2011 ; Klotoé et al., 2013) ont été conduites chez 100 consommateurs de légumes-feuilles, 30 vendeuses de légumes-feuilles des marchés et 10 tradithérapeutes des communes d'Abomey-Calavi et de Cotonou. Au total, une population de 140 individus a été enquêtée. La période d'étude s'est étalée de février à juin 2014.

L'enquête chez les vendeuses et les consommateurs de légumes-feuilles s'est déroulée dans douze marchés de ces deux communes. Le critère de choix des vendeuses de légumes-feuilles a résidé essentiellement dans la richesse de leur étalage, suivant une échelle d'au moins cinq (05) différents types de légumes feuilles par étalage. L'approche des vendeuses de légumes feuilles était basée sur le dialogue en langue locale (Fon, Goun, Mina ou Mahi), accompagnée de l'achat des légumes-feuilles thérapeutiques de grande 
consommation vendues dans le traitement des diarrhées.

Les consommateurs quant à eux, étaient choisis de façon aléatoire parmi les clients des vendeuses de légumes feuilles dans les marchés et interviewés selon la même approche que chez les vendeuses.

L'enquête chez les tradithérapeutes a été une enquête de vérification ou de confirmation des données ethnobotaniques recueillies cher les vendeuses et les consommateurs. Elle était basée sur des interviews individuelles en langues locales. Cette enquête a été précédée d'une phase préparatoire au cours de laquelle des rencontres ont été organisées pour échanger avec les tradithérapeutes sur les objectifs de l'étude, la méthode à utiliser, les résultats attendus et les bénéfices qui pourraient en découler pour toute la population. Ces échanges ont permis d'obtenir le consentement éclairé des tradithérapeutes.

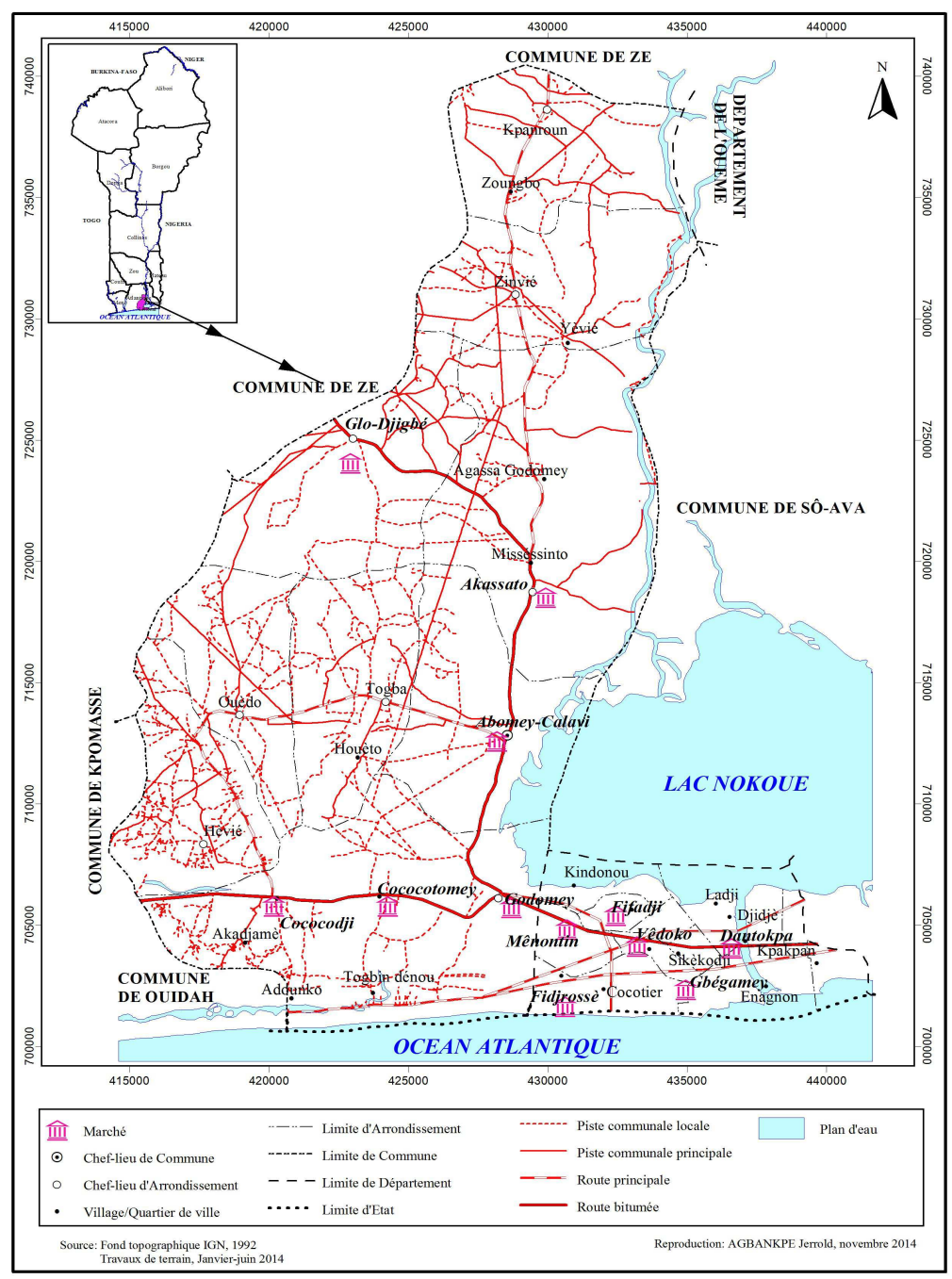

Figure 1 : Localisation des marchés visités dans les communes de Cotonou et d'Abomey-Calavi. 
Les informations recueillies de toutes ces enquêtes ont concerné les caractéristiques socioculturelles des personnes ressources (âge, sexe, niveau d'étude, revenus mensuels, etc....) et les données ethnobotaniques telles que les noms locaux, les organes végétaux utilisés, les maladies traitées et les types morphologiques. L'approche bibliographique a permis de compléter ces informations à partir de documents ethnobotaniques (Akoègninou, 2004 ; Dansi et al., 2008).

Les légumes feuilles cités par les enquêtés ont été aussitôt récoltés et échantillonnés. L'identification taxonomique a été réalisée à l'Herbier National du Bénin de l'Université d'Abomey-Calavi par le botaniste co-auteur de ce manuscrit.

\section{Traitement des données}

Les données enregistrées sur les fiches d'enquête ont été ensuite saisies et analysées par le logiciel SPSS 17.0. La fréquence de citation ( $\mathrm{Fc}$ de chaque plante a été déterminée par la formule :

$F C=\frac{\text { Nombre de citations pour le légume feuille considéré }}{\text { Nombre total de citations pour tous les légumes feuilles }} \times 100$

\section{RESULTATS}

\section{Caractéristiques socioculturelles des enquêtés}

$\mathrm{Au}$ total, cette étude a porté sur 140 personnes dont $74 \%$ de sexe féminin contre $26 \%$ de sexe masculin. L'âge moyen des enquêtés est de cinquante ans (entre 25 et 75 ans). Les sujets d'au moins 45 ans représentent plus de la moitié de la population d'étude (Figure 2). De même, plus de la moitié de la population d'étude a un niveau d'étude inférieur au secondaire (Figure 3). $54 \%$ des enquêtés ont un revenu mensuel entre le Salaire Minimum Interprofessionnel de Croissance (SMIC) et 40000 FCFA (Figure 4). De plus, 87,86\% des enquêtés affirment avoir hérité des connaissances médicinales des légumes feuilles dans le traitement des maladies.

\section{Données ethnobotaniques}

Les données issues de ces enquêtes ont permis de recenser vingt-sept (27) espèces de légumes feuilles regroupées en vingt-trois (23) genres et appartenant à dix-sept (17) familles botaniques. Les plus représentées sont les Amaranthaceae et les Lamiaceae (Figure 5). En considérant le type morphologique, les Herbes sont les plus fréquentes avec un pourcentage de $51,85 \%$. Elles sont suivis des Arbustres (40,74\%), des Lianes et des Arbres avec un même pourcentage de $03,70 \%$. Sur les 27 espèces recensées, 11 sont cultivées et 16 sont à l'état sauvage ou spontané (Tableau 1). Tous ces légumes feuilles sont préparés à l'état frais ou séchés (poudres) et consommés comme légume d'accompagnement (sauce) ou utilisés comme tisane dans le traitement des diarrhées. De plus, certains d'entre eux sont encore utilisés pour traiter d'autres maladies (Tableau 1).

En tenant compte du nombre total de citations de légumes feuilles consommés ou utilisés pour le traitement des diarrhées, l'espèce Ocimum gratissimum L.est citée avec un pourcentage de $29,05 \%$.

Ensuite, viennent Vernonia amygdalina Delile.(16,80\%), Crateva adansonii DC ssp adansonii $(13,49 \%)$ et Sesamum radiatum L. $(11,41 \%)$ (Tableau 1). 


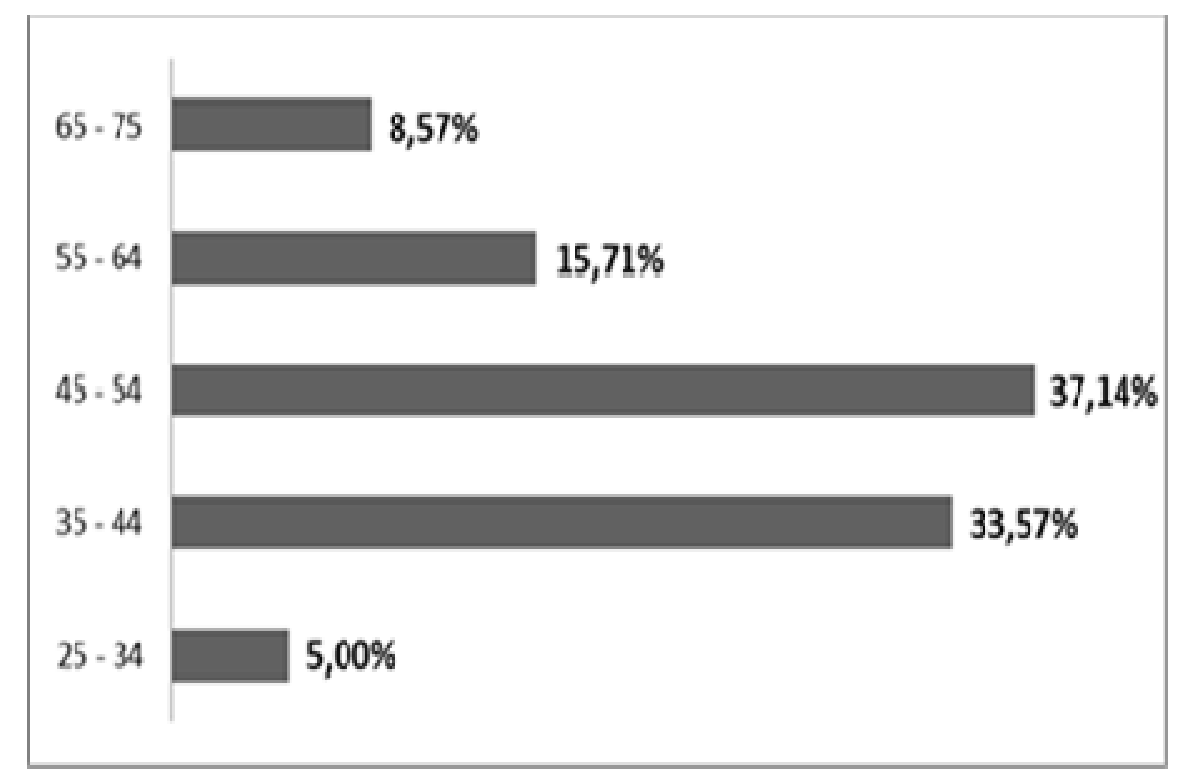

Figure 2 : Distribution des enquêtés selon l'âge.

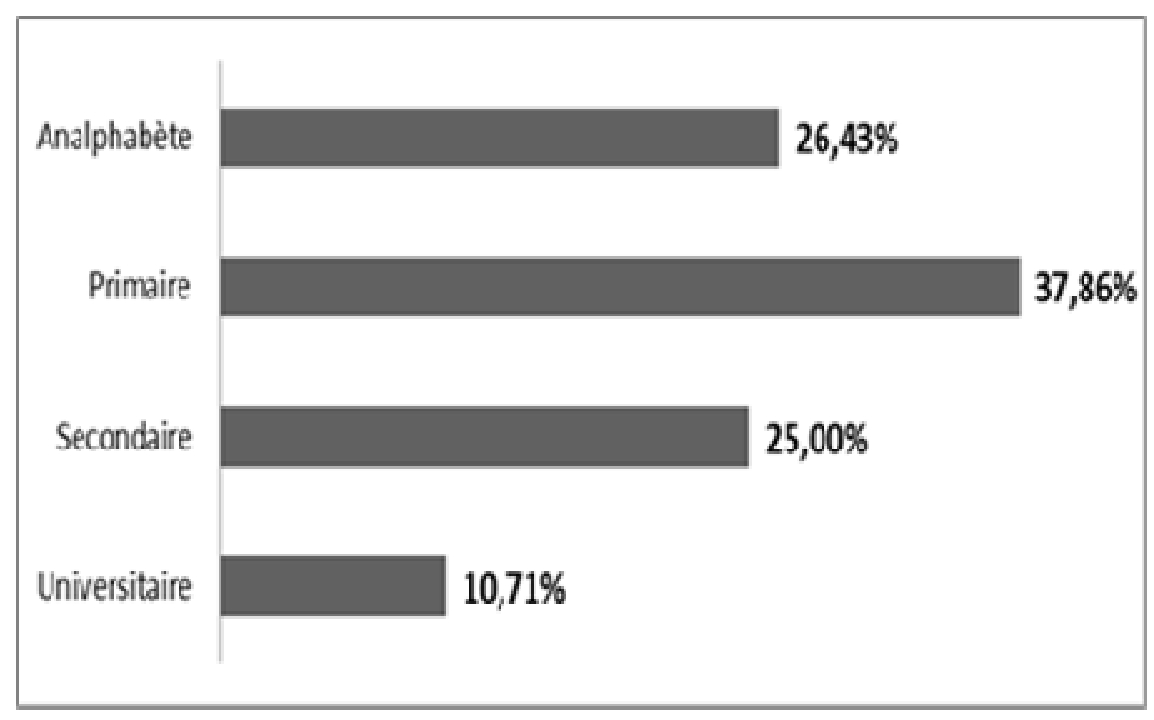

Figure 3 : Distribution des enquêtés selon le niveau d'étude. 


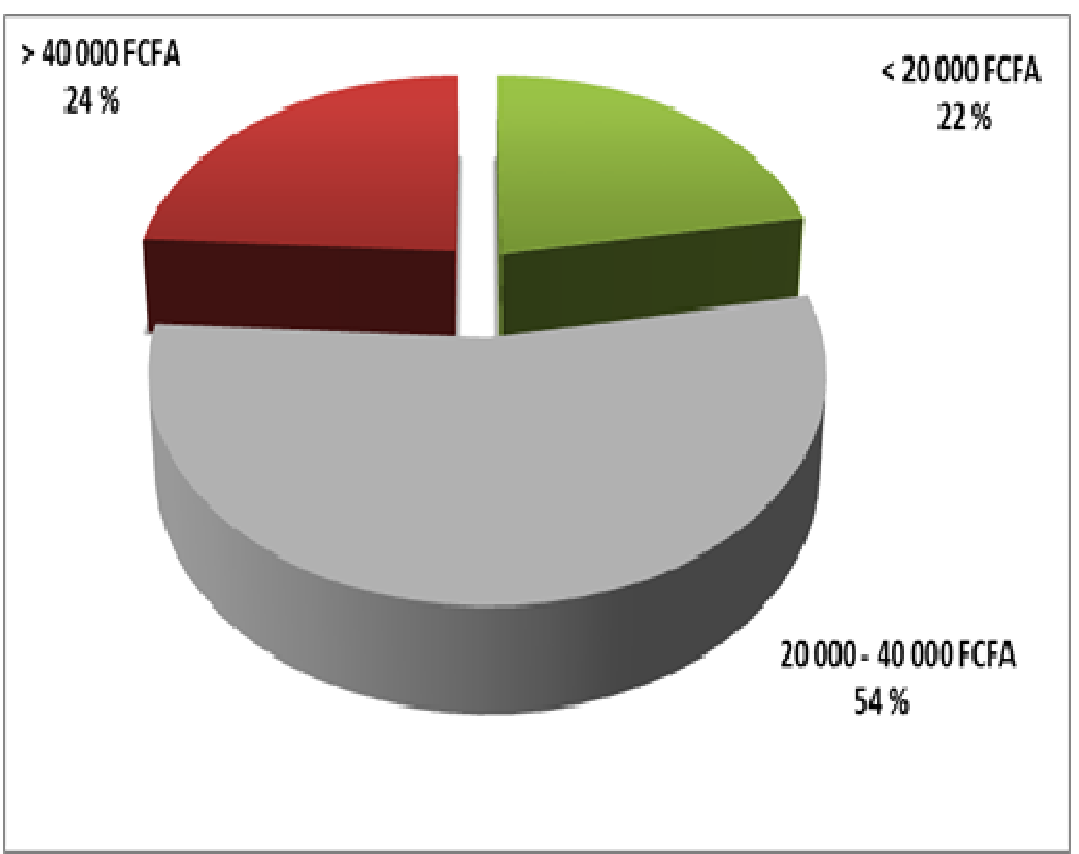

Figure 4 : Fréquence des revenus mensuels des consommateurs de légumes-feuilles.

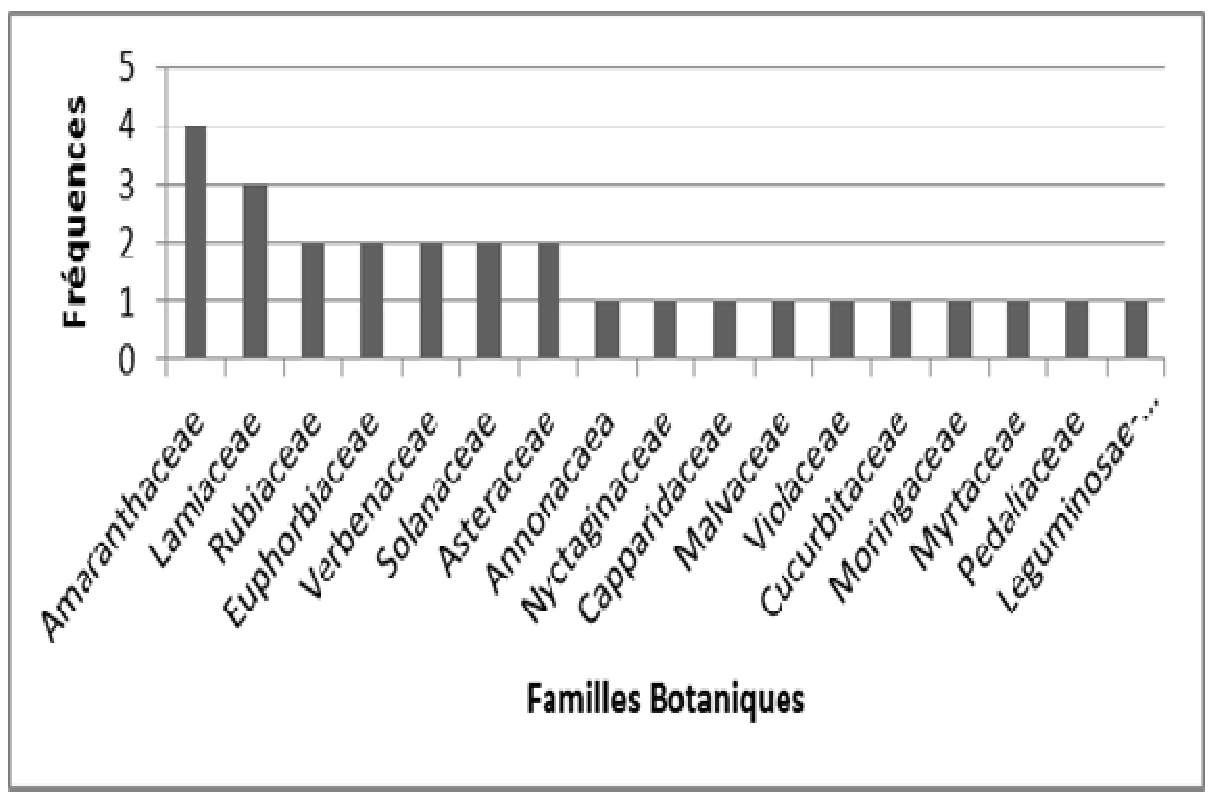

Figure 5 : Fréquence des familles botaniques. 
Tableau 1 : Principaux légumes feuilles consommés et utilisés au sud-Bénin pour le traitement des diarrhées.

\begin{tabular}{|c|c|c|c|c|c|c|c|c|}
\hline $\mathbf{N}^{\circ}$ & Espèces & Noms de familles & T M & Statut & Org U & Noms vernaculaires & Vertus médicinales & Fc \\
\hline 1 & Ocimum gratissimum $\mathrm{L}$. & Lamiaceae & $\mathrm{H}$ & $\mathrm{C}$ & $\mathrm{F}$ & Tchiayo & $\begin{array}{l}\text { Maux de ventre } \\
\text { Diarrhées, Dysenterie } \\
\text { Hypertension, } \\
\text { Candidoses }\end{array}$ & $29,05 \%$ \\
\hline 2 & $\begin{array}{l}\text { Vernonia amygdalina } \\
\text { Delile. }\end{array}$ & Asteraceae & $\mathrm{Ar}$ & $\mathrm{C}$ & $\mathrm{F}$ & Amanvivè $^{1 ; 2}$ & $\begin{array}{l}\text { Diarrhées, Constipation, } \\
\text { Vermifuge }\end{array}$ & $16,80 \%$ \\
\hline 3 & Crateva adansonii DC. & Capparidaceae & $\mathrm{Ar}$ & $\mathrm{S}$ & $\mathrm{F}$ & Hontonzuzoué $^{1 ; 4}$ & $\begin{array}{l}\text { Diarrhées, Abcès, } \\
\text { Paludisme, Constipation, } \\
\text { Hypertension }\end{array}$ & $13,49 \%$ \\
\hline 4 & Sesamum radiatum $\mathrm{L}$. & Pedaliaceae & $\mathrm{H}$ & $\mathrm{C}$ & $\mathrm{F}$ & $\mathrm{Akanmanku}^{1} ; \mathrm{Agbô}^{2}$ & $\begin{array}{l}\text { Diarrhées, Délivrance } \\
\text { chez la femme enceinte }\end{array}$ & $11,41 \%$ \\
\hline 5 & Moringa oleifera Lam. & Moringaceae & $\mathrm{Ar}$ & $\mathrm{C}$ & $\mathrm{F}$ & Kpatchi $^{3} ;$ Kpatiman $^{1}$ & $\begin{array}{l}\text { Diarrhées, Fièvre, } \\
\text { Céphalées, Convulsion }\end{array}$ & $07,88 \%$ \\
\hline 6 & Solanum macrocarpon L. & Solanaceae & $\mathrm{H}$ & $\mathrm{C}$ & $\mathrm{F}$ & Gboma $^{1 ; 2 ; 3}$ & Diarrhées, Paludisme & $05,19 \%$ \\
\hline 7 & Centrostachys aquatica & Amaranthaceae & $\mathrm{H}$ & $\mathrm{S}$ & $\mathrm{F}$ & Toloman $^{8}$ & Diarrhées & $03,32 \%$ \\
\hline 8 & Amaranthus cruentus L. & Amaranthaceae & $\mathrm{H}$ & $\mathrm{C}$ & $\mathrm{F}$ & Fortètè $^{1 ; 2}$ & Diarrhées, Maux de & $02,28 \%$ \\
\hline
\end{tabular}


A. J. AGBANKPE et al. / Int. J. Biol. Chem. Sci. 8(4): 1784-1795, 2014

\begin{tabular}{|c|c|c|c|c|c|c|c|c|}
\hline & & & & & & & ventre & \\
\hline 9 & Vitex doniana Sweet. & Verbenaceae & A & $\mathrm{S}$ & $\mathrm{F}$ & Fonman $^{1 ; 2 ; 3}$ & $\begin{array}{ll}\text { Diarrhées, } & \text { Fièvre, } \\
\text { Rougeole, } & \text { Ulcère } \\
\text { intestinal } & \end{array}$ & $01,66 \%$ \\
\hline 10 & Psidium guajava $\mathrm{L}$. & Myrtaceae & $\mathrm{Ar}$ & $\mathrm{S}$ & $\mathrm{F}$ & Gouagbéman $^{6}$ & $\begin{array}{l}\text { Diarrhées, } \quad \text { Ictère, } \\
\text { Dysenterie }\end{array}$ & $01,04 \%$ \\
\hline 11 & $\begin{array}{l}\text { Hybanthus enneaspermus } \\
\text { L. }\end{array}$ & Violaceae & $\mathrm{H}$ & $\mathrm{S}$ & $\mathrm{F}$ & Gbogokou $^{9}$ & Diarrhées, Dystocies & $01,04 \%$ \\
\hline 12 & $\begin{array}{l}\text { Macrosphyra longistyla } \\
\text { DC. }\end{array}$ & Rubiaceae & $\mathrm{Ar}$ & $\mathrm{S}$ & $\mathrm{F}$ & Azinguidigokoun $^{4}$ & $\begin{array}{l}\text { Diarrhées, Constipation, } \\
\text { Dysenterie }\end{array}$ & $0,83 \%$ \\
\hline 13 & Solanum aethiopicum & Solanaceae & $\mathrm{H}$ & $\mathrm{C}$ & $\mathrm{F}$ & Gbognanmain $^{1 ; 3}$ & $\begin{array}{l}\text { Diarrhées, Paludisme, } \\
\text { Maux de ventre }\end{array}$ & $0,83 \%$ \\
\hline 14 & Vernonia colorata Willd. & Asteraceae & $\mathrm{Ar}$ & $S$ & $\mathrm{~F}$ & Gblé $^{1 ; 2}$ & $\begin{array}{l}\text { Diarrhées, } \quad \text { Stimule } \\
\text { l'appétit }\end{array}$ & $0,83 \%$ \\
\hline 15 & Ocimum americanum $\mathrm{L}$. & Lamiaceae & $\mathrm{H}$ & $\mathrm{S}$ & $\mathrm{F}$ & Akohun $^{1 ; 3}$ & $\begin{array}{l}\text { Diarrhées, Vomissement, } \\
\text { Affection d'yeux }\end{array}$ & $0,62 \%$ \\
\hline 16 & $\begin{array}{l}\text { Pandiaka invalucrata } \\
\text { Moq. }\end{array}$ & Amaranthaceae & $\mathrm{H}$ & $\mathrm{S}$ & $\mathrm{F}$ & Gassalantoro $^{10}$ & Diarrhées & $0,62 \%$ \\
\hline 17 & Aerva lanata $\mathrm{L}$. & Amaranthaceae & $\mathrm{H}$ & $\mathrm{S}$ & $\mathrm{F}$ & Ipofi $^{10}$ & $\begin{array}{l}\text { Diarrhées, Affection } \\
\text { oculaire }\end{array}$ & $0,41 \%$ \\
\hline
\end{tabular}


A. J. AGBANKPE et al. / Int. J. Biol. Chem. Sci. 8(4): 1784-1795, 2014

\begin{tabular}{|c|c|c|c|c|c|c|c|c|}
\hline 18 & Momordica charantia $\mathrm{L}$. & Cucurbitaceae & $\mathrm{L}$ & $\mathrm{S}$ & $\mathrm{F}$ & Gnisinkin $^{2} ;$ Voï $^{3}$ & $\begin{array}{l}\text { Diarrhées, Maux de } \\
\text { ventre }\end{array}$ & $0,41 \%$ \\
\hline 19 & Annona senegalensis Pers & Annonaceae & $\mathrm{Ar}$ & $\mathrm{S}$ & $\mathrm{F}$ & Nyiglwe $^{1}$ Nyankéklé $^{7}$ & $\begin{array}{l}\text { Diarrhées, Maux de } \\
\text { ventre }\end{array}$ & $0,41 \%$ \\
\hline 20 & $\begin{array}{l}\text { Chassalia kolly } \\
\text { Schumach. }\end{array}$ & Rubiaceae & $\mathrm{Ar}$ & $\mathrm{S}$ & $\mathrm{F}$ & Djètinman $^{1}$ & Diarrhées, Diabète & $0,41 \%$ \\
\hline 21 & $\begin{array}{l}\text { Lippia multiflora } \\
\text { Moldenke. }\end{array}$ & Verbenaceae & $\mathrm{H}$ & $\mathrm{S}$ & $\mathrm{F}$ & Agalala $^{1}$ & Diarrhées, Dysenterie & $0,21 \%$ \\
\hline 22 & Hoslundia opposite Vahl. & Lamiaceae & $\mathrm{Ar}$ & $\mathrm{S}$ & $\mathrm{F}$ & Agbanlidôgbo $^{4}$ & $\begin{array}{l}\text { Diarrhées, Maux de } \\
\text { ventre }\end{array}$ & $0,21 \%$ \\
\hline
\end{tabular}

Noms vernaculaires : 1-Fon ; 2-Mahi ; 3-Adja ; 4-Cotafon ; 5-Toli ; 6-Péda ; 7-Goun ; 8-Oumaingbé ; 9-Bariba ; 10-Ani ; T M = Types Morphologiques : A-arbre ; Ar-arbuste ; -liane ; Statut : C-cultivé ; Sétat sauvage ; Org U = Organes végétaux Utilisés : F-feuilles ; Fc = Fréquence de citation de chaque légume. 


\section{DISCUSSION}

Les résultats issus de cette étude ont montrés que les enquêtés sont majoritairement de sexes féminins et âgés d'au moins cinquante ans. Cela s'explique par le fait qu'au Bénin, c'est la femme qui par excellence s'occupe de la vente des légumes feuilles dans les marchés et surtout de la cuisine quand on parle de consommation de légumes feuilles. Il a été surtout admis qu'en Afrique, ce sont les sages, c'est-à-dire les personnes âgées qui détiennent la connaissance traditionnelle de traitement des maladies. De plus, les vertus médicinales des légumes feuilles ou des plantes sont des connaissances ancestrales qui se transmettent de génération en génération (Klotoé et al., 2013 ; Dassou et al., 2014). 38,57\% des enquêtés sont âgés de moins de 45 ans, donc relativement jeunes. Ce qui s'explique par le fait que c'est souvent toutes les connaissances médicinales traditionnelles qui sont transmisses des ascendants (sages) aux descendants (jeunes) à telle enseigne que les deux types d'individus ont pratiquement le même niveau de connaissance (Dassou et al., 2014). Ceci est d'autant plus justifiable que $87,86 \%$ des personnes enquêtées ont signalé avoir hérité ces connaissances médicinales des légumes feuilles de la famille. C'est dans cette même optique que Tamboura et al. (1998) ont signalé que l'ethnomédecine traditionnelle est une science de la société et leur contenu demeure un patrimoine familial. 64,29\% des enquêtés ont un niveau d'étude inférieur au secondaire et $35,71 \%$ ont fait au moins le secondaire. Ces résultats montrent que l'usage médicinal des légumes feuilles ou leur consommation n'est pas seulement l'apanage des illettrés (populations rurales) mais de tout le monde. $54 \%$ des consommateurs de légumes feuilles, ont un revenu mensuel entre 20000 FCFA et le SMIC sans négligé les $24 \%$ qui ont un revenu supérieur au SMIC.

Ces résultats montrent que la consommation ou l'utilisation des légumes feuilles pour le traitement des diarrhées n'est pas seulement l'apanage des pauvres, mais de toutes les couches sociales.
Les 27 espèces végétales à usages médicinales recensées représentent $0,96 \%$ de la flore totale du Bénin estimée à 2807 espèces (Akoégninou et al., 2006) et $14,44 \%$ des espèces de légumes feuilles traditionnels consommés au Bénin estimée à 187 espèces (Dansi et al., 2008). Les familles d'espèces botaniques les plus représentées sont celles des Amaranthaceae $(23,53 \%)$ et des Lamiaceae (17,65\%). Ces résultats corroborent ceux d'Omara-Achong et al. (2012) au Nigéria. Par contre, ils sont contraires à ceux présentés par Dansi et al. (2008). En effet, ces derniers ont, au cours de leur étude, montré que c'est plutôt la famille des Asteraceae et des Cucurbitaceae qui étaient les plus représentées. Mais curieusement, les pourcentages de représentativité des Amaranthaceae (23,07\%) et des Lamiaceae $(11,54 \%)$ dans le travail de Dansi et al. (2008) sont relativement conformes aux nôtres. Cet état de choses est compréhensible car la zone géographique et ethnique diffère selon les deux études, l'un concernant uniquement le Sud-Bénin alors que l'autre prenait en compte tout le territoire national d'où la dominance des Asteraceae et des Cucurbitaceae, mais le taux de représentativité des Amaranthaceae et des Lamiaceae reste approximativement le même. La réalité en ce qui concerne la taxonomie des espèces de légumes feuilles consommés est donc différente selon la zone géographique et ethnique. De plus, la présente étude concerne strictement les espèces de légumes feuilles consommés et utilisés pour le traitement des diarrhées alors que celle de Dansi et al. (2008) concerne l'inventaire de tous les espèces de légumes feuilles traditionnels consommés au Bénin. Parmi les espèces recensées, 40,74\% sont des arbustes et $51,85 \%$ des herbes. Cela ne corrobore pas les résultats de Dansi et al. (2008) qui ont présenté un pourcentage de $19,78 \%$ des arbustes contre $64,78 \%$ des herbes. Au nombre des 27 espèces de légumes feuilles recensées, 11, soit 40,74\% sont cultivées dans les champs ou dans les jardins maraîchers et $16(59,26 \%)$ poussent à l'état sauvage. ces résultats sont conformes à ceux 
de Dansi et al. (2008) dans le sens où il y a une dominance des espèces sauvages (47 espèces cultivées contre 140 à l'état sauvage). Ce qui montre que dans beaucoup de régions au Bénin, le mode de vie est toujours traditionnel et que l'alimentation ou la santé des populations reste encore dépendante de la cueillette. Cependant, ces résultats sont contraires à ceux d'Omara-Achong et al. (2012) où $74,43 \%$ des légumes feuilles traditionnels consommés dans les villages d'Ogoja et Cala Bar au Nigéria étaient cultivés contre $25,57 \%$ à l'état sauvage.

Parmi les légumes feuilles thérapeutique consommés ou utilisés comme tisane dans le traitement des diarrhées, Ocimum gratissimum L. est très utilisé. Elle est citée à 29,05\% dans les 482 citations recensées. Elle est très efficace pour traiter les diarrhées, ce qui confirme les travaux d'Adebolu et Oladimeji (2005) au Nigéria ou l'efficacité antibactérienne des extraits d'Ocimum gratissimum L. sur les bactéries responsables des diarrhées a été montrée. Vernonia amygdalina Delile. (16,80\%), Crateva adansonii DC. ssp. adansonii. $(13,49 \%)$ et Sesamum radiatum L. $(11,41 \%)$ sont aussi moyennement citées. Ces trois espèces de légumes feuilles comme Ocimum gratissimum L. sont consommés ou utilisés comme tisane pour traiter les infections diarrhéiques. Mais dans le même temps, elles sont utilisés pour traiter plusieurs d'autres maladies comme l'ont souligné plusieurs auteurs béninois (Akoégninou et al., 2006 ; Dansi et al., 2008).

\section{Conclusion}

Cette étude a souligné le rôle essentiel des tradithérapeutes dans le traitement des diarrhées au Bénin. L'étude ethnobotanique réalisée sera très utile pour les scientifiques en vue d'études ultérieures. Ces études permettront d'une part d'évaluer les propriétés antibactériennes et anti-diarrhéiques et d'autre part, d'isoler et d'identifier des principes actifs qui pourraient donner lieu à des médicaments anti-diarrhéiques pour le bienêtre de la population.

\section{REFERENCES}

Adebolu TT, Oladimeji SA. 2005. Antimicrobial activity of leaf extracts of Ocimum gratissimum on selected diarrhoea causing bacteria in southwestern Nigeria. African Journal of Biotechnology, 4(7): 682-684.

Akoègninou A. 2004. Recherches botaniques et écologiques sur les forêts actuelles du Bénin. Thèse d'Etat, Université de Cocody-Abidjan, Côte d'Ivoire, p. 326.

Bryce J, Boschi-Pinto C, Shibuya K. 2005. WHO estimates of the causes of death in children. Lancet, 365: 1147-1152.

Dansi A, Adjatin A, Vodouhè R, Adéoti K, Adoukonou-Sagbadja H, Faladé V, Yédomonhan $\mathrm{H}$, Akoégninou A, Akpagana K. 2008. Biodiversité des Légumes Feuilles Traditionnels Consommés au Bénin. Bibliothèque Nationale du Bénin : Benin; 174.

Dassou HG, Ogni CA, Yédomonhan $\mathrm{H}$, Adomou AC, Tossou M, Dougnon JT, Akoègninou A. 2014. Diversité, usages vétérinaires et vulnérabilité des plantes médicinales au Nord-Bénin. Int. J. Biol. Chem. Sci., 8(1): 189-210.

Dibong SD, Mpondo Mpondo E, Ngoye A, Kwin MF, Betti JL. 2011. Ethnobotanique et phytomédecine des plantes médicinales de Douala. J. Appl. Biosci., 37: 2496-2507.

Dossou ME, Houessou GL, Lougbégnon OT, Tenté AH, Codjia JT. 2012. Ethnobotanical Study of Ligneous Species in Agonvè Swampy Forest and Surrounding Area in Benin. Tropicultura, 30(1): 41-48.

Fah L, Klotoé JR, Dougnon V, Koudokpon H, Fanou VB, Dandjesso C, Loko F. 2013. Étude ethnobotanique des plantes utilisées dans le traitement du diabète chez les femmes enceintes à Cotonou et Abomey-Calavi (Bénin). Journal of Animal \& Plant Sciences, 18(1): 26472658.

Gagnon M. 2007. Rôle des probiotiques lors d'infections entériques d'origine bactérienne et virale : analyses in vitro et 
études in vivo chez des modèles murins. $\mathrm{PhD}$ thèse, Université Laval, Québec, p. 170.

INSAE. 2013. Recensement Général de la Population et de l'Habitat. MDAEP, INSAE : Cotonou, Bénin ; 8.

Klotoé JR, Dougnon TV, Koudouvo K, Atègbo JM, Loko $\mathrm{F}$, Akoègninou $\mathrm{A}$, Aklikokou K, Dramane K, Gbeassor M. 2013. Ethnopharmacological survey on antihemorrhagic medicinal plants in South of Benin. European Journal of Medicinal Plants, 3(1): 40-51.

Liu L, Johnson HL, Cousens S, Perin J, Scott S, Lawn JE, Rudan I, Campbell H, Cibulskis R, Li M, Mathers C, Black RE. 2012. Global, regional, and national causes of child mortality: an updated systematic analysis for 2010 with time trends since 2000. Lancet, 379: 21512161.
Omara-Achong TE, Edwin-Wosu NL, Edu EA, Nkang AE. 2012. Survey of indigenous vegetables species in parts of Ogoja and Cala Bar, Cross River State, Nigeria. Euro. J. Exp. Bio., 2(4): 12891301.

OMS. 2011. Statistiques sanitaires mondiales. OMS, p. 171.

Sinha NK. 2011. Handbook of Vegetables and Vegetables Processing. Blackwell Publishing Ltd: England; 788.

Tamboura H, Kaboré H, Yaméogo SM. 1998. Ethnomédecine vétérinaire et pharmacopée traditionnelle dans le plateau central du Burkina Faso : cas de la province du Passoré. Biotechnol. Agron. Soc. Environ., 2(3): 181-191.

WHO. 2009. Traditional medicine; vailable online from http://www.who.int/topics/ tradidional_medicine /en/ Accessed on April 2014. 